Check for updates

Cite this: RSC Adv., 2018, 8, 37254

\title{
The influence of the morphological characteristics of nanoporous anodic aluminium oxide (AAO) structures on capacitive touch sensor performance: a biological application $\uparrow$
}

J. O. Carneiro, (DD ${ }^{* a}$ F. Machado, ${ }^{a}$ M. Pereira, ${ }^{a}$ V. Teixeira, ${ }^{a}$ M. F. Costa, ${ }^{a}$ Artur Ribeiro, ${ }^{b}$ Artur Cavaco-Paulo (D) ${ }^{b}$ and A. P. Samantilleke ${ }^{\star a}$

\begin{abstract}
This work is devoted to the study of the influence of different anodic aluminium oxide (AAO) morphologies on the sensitivity and performance of an AAO-based capacitive touch sensor. The AAO structures were fabricated in a cylindrical homemade anodization cell made from a solid polycarbonate billet via a lathe machining process. The AAO morphologies were obtained from the anodization of Al foil by using three different types of electrolyte (sulphuric acid, oxalic acid and phosphoric acid) and their morphologies are reported and compared using scanning electron microscopy (SEM) micrographs and current-time characteristic curves. The sensors were fabricated by integrating the AAO structure with a nanotextured gold thin film deposited over the AAO layer by thermal evaporation, thus realizing a type of metal/insulator/metal parallel-plate capacitance sensor. It is demonstrated that AAO morphologies have influence on the performance of the AAO-based capacitive touch sensors. The variation of the capacitance of the sensors is investigated in this work for the AAO structures produced from anodization in an attempt to select anodizing conditions for a biological application aiming to detect small microorganisms such as bacterial colonies of Escherichia coli.
\end{abstract}

Received 7th September 2018 Accepted 29th October 2018

DOI: $10.1039 / c 8 r a 07490 a$

rsc.li/rsc-advances is noteworthy that almost a century after the observation of $\mathrm{Al}$ anodizing, this phenomenon has recently triggered interest and acquired increased importance to the scientific community that works in leading scientific areas such as the field of nanotechnology. This trajectory was commenced with the work developed by Masuda and Fukuda, ${ }^{3}$ who in 1995, reported the production of porous AAO layers exhibiting a self-ordered pore structure and one year later Masuda and $\mathrm{Satoh}^{4}$ developed, what is known as two-step anodizing process by which porous AAO could be obtained with improved structural periodicities over larger spatial areas. AAOs find many high-tech applications in nanostructures and devices. Among different research areas, the use of AAO in one-dimensional nanostructures is noteworthy, ${ }^{5-11}$ as tubular membranes for hemodialysis, ${ }^{12,13}$ membranes for gas separation, drug delivery and bone fixation $^{14-17}$ energy storage devices, among others. ${ }^{18-21}$ Recent work by several authors ${ }^{12,14}$ prove that nanostructured functional layers of significantly enlarged surface areas for sensor applications can be made from thin porous alumina films.

The fabrication of AAO is based on simple, cost-effective selfordering anodization of aluminium, which yields vertically aligned, highly ordered nanoporous structures.

It is well known that there are two main processes for AAO fabrication, namely mild anodization (MA) and hard anodization (HA)., ${ }^{3,22}$ In general both of these processes utilize three different types of acidic electrolytes, namely sulphuric, oxalic,
${ }^{a}$ Centre of Physics, University of Minho, Azurém Campus, 4800-058 Guimarães, Portugal.E-mail: carneiro@fisica.uminho.pt; anura@fisica.uminho.pt

${ }^{b}$ Centre of Biological Engineering, University of Minho, Gualtar Campus, 4710-057 Braga, Portugal

$\dagger$ Electronic supplementary information (ESI) available. See DOI 10.1039/c8ra07490a 
and phosphoric acids. The MA process has been mostly used in academic work for a wide variety of nanotech applications. However, since MA uses a low anodizing potential, the growth rate of AAO nanostructures is in general slow, and the process becomes even more time consuming under the two-step anodizing conditions. On the contrary, HA is mostly used by certain industrial applications due to efficient growth of AAO nanostructures and improved periodicity of nanopores over larger spatial areas. HA process requires the application of relatively high anodic voltages, thus leading to a high current density, which causes the HA process to have exothermic characteristics (as the result of Joule's heating). However, the generation of excessive heat during HA processes is also responsible for the acidic dissolution of the formed AAO nanoporous structure as well as strongly contributing to the occurrence of local breakdown, ${ }^{23}$ for which reason the fabrication of AAO nanostructures under HA conditions has not been widely used in nanotechnology applications. Several approaches have been made to find effective solutions to extract the reaction heat such as the use of cooling systems ${ }^{\mathbf{2 4}}$ or certain cooling reagents (e.g. ethanol is used as coolant agent) added to the aqueous electrolytes in order to compensate or minimize the generated heating and local burning problems. ${ }^{25,26}$ In recent years, capacitive type tactile or pressure sensors have been reported..$^{27,28}$ Hong et al. ${ }^{29}$ used AAO structures to serve as template to form a nanotextured gold film to fabricate a capacitive touch sensor to detect small contact forces, such as insects. The AAO structures were formed by two-step anodizing conditions from an aqueous solution of $0.3 \mathrm{M}$ oxalic acid at a voltage of $47 \mathrm{~V} \mathrm{DC}$. According to the authors, they obtained an AAO structure with an average nanopore diameter of $54 \mathrm{~nm}$ and a porosity of about $23.6 \%$. After covering the entire pore structure by a nanotextured Au top electrode, they registered a capacitance of $\sim 1.75 \mathrm{nF}$ and an average capacitance variation of $4.1 \%$.

Taking as basis the study performed by Hong et al., we have performed a experimental campaign in which the main objective and novelty is to study the influence of different AAO morphologies, obtained by using three different acid electrolytes and anodization conditions, on the sensitivity and performance of an AAO-based capacitive touch sensor. The sensor fabricated proved to be able to detect the surface contact interaction of small objects (bacterial colonies of Escherichia coli and also a tiny metal plate were used in this work), due to variations on the nanotextured Au electrode area. Escherichia coli (E. coli) is particularly interesting in this study as capacitive sensors of this nature generally show high selectivity and is able to distinguish $E$. coli when present together with competing bacterial strains, which are known to have similar shape. ${ }^{28}$ Idil et al. also reported suitability of similar capacitive touch sensors for the detection of $E$. coli, having investigated selectivity of the E. coli-imprinted electrode with other bacterial strains such as Staphylococcus aureus (S. aureus), Bacillus subtilis (B. subtilis), and Salmonella paratyphi (S. paratyphi) using real-time detection experiments. ${ }^{30}$

The performance of the AAO capacitive-type sensor and its dependence on the AAO morphology, which is characterized by different structural parameters such as inter-pore distance
$\left(D_{\text {int }}\right)$, pore diameter $\left(D_{\mathrm{p}}\right)$ and porosity $(P)$, which in turn, are dependent on the anodizing conditions, namely the electrolyte type, concentration, temperature, applied anodizing potential and anodizing time will be investigated in this work.

\section{Sensing principle and mechanism of the AAO capacitive-type sensor}

The underlying physical principle that governs the practical performance of the AAO capacitive sensor, is associated with the variation of capacitance, which results from changes on the AAO surface area (due to the contact with the object) and also on the properties of the dielectric medium. Under the scope of this work, the presence of small objects was simulated via the use of a specific bacterial culture medium that clearly demonstrated a strong capability of the as-fabricated AAO-based capacitive device to be used in biomedical applications, such as in hypothetical scenarios where someone, over time, intends to monitor the progression stage of a particular type of bacterial disease.

Fig. 1 refers to the underlying concept of a nanoporous AAO capacitive sensor. The AAO structure is grown through the anodization process from a pure aluminium substrate. The structural properties (i.e. $D_{\text {int }}$ and $P$ ), of the obtained AAO structures depends on the type of electrolytes used, namely sulphuric, oxalic, and phosphoric acids.

Analysing Fig. 1(a), it is possible to identify the nanotextured top electrode, which is composed of an Au thin film directly deposited on the AAO porous layer by thermal resistive evaporation. The evaporation parameters are presented later in the text. Fig. 1(b) depicts the metal-insulator-metal (MIM) capacitor device, which consists of a base metal ( $\mathrm{Al}$ ) substrate, an insulator (AAO layer) and a top metallic electrode (Au). The sensor operating mechanism can be briefly described as follows: due to the AAO nanoporous structure, which may have different structural properties according the type of used electrolyte, any external object (in this work, bacteria and also an $\mathrm{Al}$ metal disk) that could enter into contact with the surface of the MIM device will cover the surface pores to some extent, thus causing a variation on the total electrical capacitance of the MIM device (see Fig. 1(c)). This occurs because, as soon as the object contacts the nanotextured surface to cover new pores, it creates additional capacitors (where the dielectric medium is air or a particular aqueous medium in the case of the bacteria culture), which adds a parallel capacitive component to the existing MIM device and thus, an increase of the total capacitance of the MIM device is expected. In this work the E. coli was used as a model microorganism to assess and validate the ability of the AAO based sensors to detect microorganisms.

\section{Experimental}

3.1 Fabrication technology: production of AAO nanoporous structures by $\mathrm{Al}$ anodization

The nanoporous AAO structures were electrochemically produced from $\mathrm{Al}$ (99.999\% purity) foils $(0.25 \mathrm{~mm}$ thick, acquired from Sigma-Aldrich) by one-step anodizing process. 


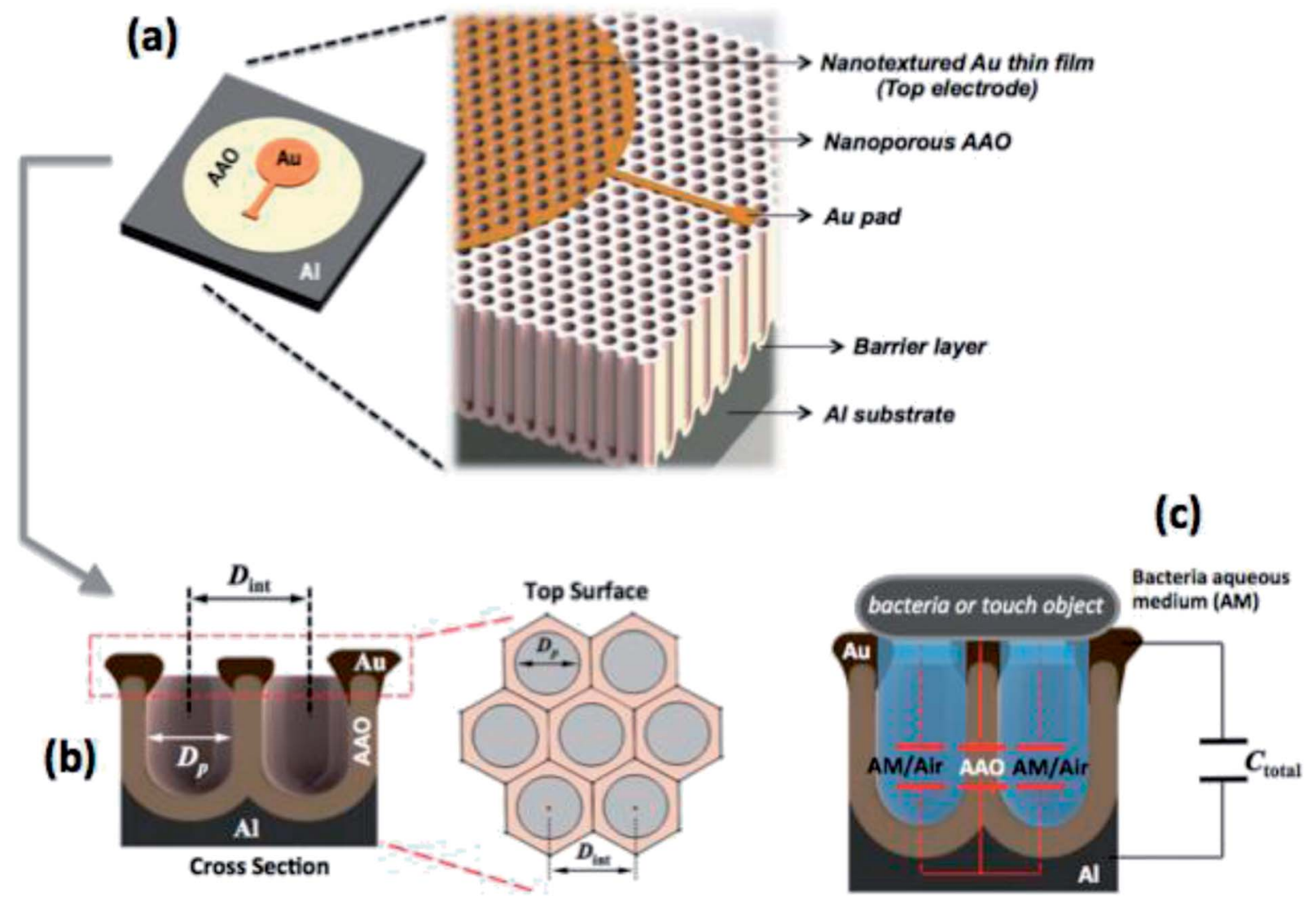

Fig. 1 Nanoporous AAO structure used as a capacitive-type sensor; (a) schematic representation of the metal-insulator-metal (MIM) sensor architecture comprising the nanotextured thin film Au top electrode, the nanoporous AAO insulator layer and the bottom Al substrate; (b) cross section and top surface of the MIM based AAO capacitive sensor showing main structural characteristics and (c) operating mechanism of the AAO base capacitive sensor when a particular object (e.g. a colony of specific bacteria) makes a contact with the surface of the MIM sensor.

The anodizing process was realized in a homemade anodization cell comprising an anode (pre-treated $\mathrm{Al}$ foil) and a cathode $\mathrm{Pb}$ plate). The body of the electrochemical cell is made of polypropylene material and has a cylindrical geometry fabricated by lathe machining a solid polycarbonate billet.

The total volume of the anodization cell is about $220 \mathrm{ml}$, where the anode is placed at the bottom and holds a circular geometry with a diameter of $20 \mathrm{~mm}$. The cathode consists of one $\mathrm{Pb}$ plate with rectangular-type geometry and dimensions of $(105 \times 15 \times 4) \mathrm{mm}$. Some geometrical details related with the homemade anodization cell are properly identified in the ESI (Fig. SI1(a) and (b)†).

After standard substrate cleaning, the Al foils were subjected to a pre-treatment by ultra-sonication in ethanol/benzine for 10 to 15 minutes at room temperature (RT). The $\mathrm{Al}$ foils were then dried and used as anodes in the anodization cell. In order to obtain AAO structures with different structural parameters, the Al foils were anodized with the three different electrolytes: sulphuric acid $(0.3 \mathrm{M})$, oxalic acid $(0.3 \mathrm{M})$ and phosphoric acid $(0.3 \mathrm{M})$. In the case of sulphuric acid and oxalic acid electrolytes, the anodizing process was realized at room temperature (RT) and we observed that the optimum anodizing potential $(U)$ to achieve the best ordering of pores are 21 and $40 \mathrm{~V}$, respectively. For the phosphoric acid electrolyte (HA conditions), the applied anodization potential was $U=150 \mathrm{~V}$ at a temperature of $0{ }^{\circ} \mathrm{C}$. All the $\mathrm{Al}$ foils were only subjected to one anodizing step by using an EA-PS 3150-04B-power supply (from Elektro Automatik). The current transients, $I(t)$, were monitored during the anodization process using a EA-UTA 12 analogue interface (from ElektroAutomatik) equipped with LabView software. The experimental conditions and the related samples' identification (sample ID) are shown in Table 1.

Table 1 Experimental conditions of anodization process

\begin{tabular}{|c|c|c|c|c|c|}
\hline Anodizing condition & Sample ID & $\begin{array}{l}\text { Electrolyte type/ } \\
\text { concentration }[\mathrm{M}]\end{array}$ & $\begin{array}{l}\text { Electrolyte } \\
\text { temperature }\left({ }^{\circ} \mathrm{C}\right)\end{array}$ & $\begin{array}{l}\text { Applied } \\
\text { voltage (V) }\end{array}$ & $\begin{array}{l}\text { Anodizing } \\
\text { time (min) }\end{array}$ \\
\hline MA & SU & $0.3 \mathrm{M} \mathrm{H}_{2} \mathrm{SO}_{4}$ & RT & 21 & 60 \\
\hline \multirow[t]{2}{*}{ HA } & $\mathrm{P} 1$ & $0.3 \mathrm{M} \mathrm{H}_{3} \mathrm{PO}_{4}$ & 0 & 150 & 0.67 \\
\hline & P2 & & & & 1.08 \\
\hline
\end{tabular}




\subsection{Deposition of a nano-textured Au top electrode}

The deposition of the Au thin film was carried out by resistive thermal evaporation. The thermal evaporating system (ESI, Fig. SI $2(\mathrm{a}) \dagger$ ) includes a deposition chamber with a volume of $3.8 \mathrm{~L}$, a primary rotary pump (with a pumping speed of $5000 \mathrm{~L}$ $\mathrm{h}^{-1}$ ) and a diffusion pump with a pumping speed of $110 \mathrm{~L} \mathrm{~s}^{-1}$. The Au thermal evaporation was carried out in order to transfer a nano-textured gold circular top electrode with a radius of $3.75 \mathrm{~mm}$ to the AAO surface and connected to a gate with a length of $5 \mathrm{~mm}$. To serve this purpose, a specific mask fabricated from an $\mathrm{Al}$ sheet, whose geometry is shown in Fig. SI2(b) and (c), $\dagger$ was used.

Taking into account the experimental conditions specified in Table 1, AAO structures with different structural parameters were obtained. The amount of $\mathrm{Au}$ wire mass used varied according to the AAO pore diameter, namely 34, 17 and $7 \mathrm{mg}$ for samples identified according to the electrolytes used in deposition, as SU (sulphuric), OX (oxalic), P1 and P2 (phosphoric), respectively. For all of the produced samples, the gold top electrode was evaporated from a $0.5 \mathrm{~mm}$ diameter $\mathrm{Au}$ wire placed in a tungsten boat (see Fig. SI2(a) $\dagger$ ).

Complete evaporation of $\mathrm{Au}$ film to the nanoporous $\mathrm{AAO}$ surface was attained by firstly evacuating the vacuum chamber to achieve a pressure of $2 \times 10^{-6}$ mbar and then applying a current of $220 \mathrm{~A}$ and a voltage of $1.2 \mathrm{~V}$ for $2 \mathrm{~min}$ to the tungsten boat.

It is important to note that each of the fabricated AAO samples were subjected to two consecutive Au thermal evaporation stages. In the first stage, the mask pattern was totally transferred to the surface of the nanoporous AAO structure. The second $\mathrm{Au}$ evaporation stage was only performed over the gate by covering the circular region of the mask by a metal disk with a $3.75 \mathrm{~mm}$ radius, (as schematized in Fig. SI3(a) of the ESI $\dagger$ ). The purpose of this procedure was to obtain an Au top electrode having a nanotextured surface and circular geometry, which in turn is directly linked to a compact gate. All the electrical connections with the Au top electrode were performed by placing a small needle on the surface of the gate pad (see Fig.$\mathrm{SI} 3(\mathrm{~b}) \dagger)$. The Al substrate, which supports the AAO structure, acts as the bottom electrode to which, a copper wire was subsequently attached using silver paint.

\subsection{Preparation of a bacteria culture as agents for touch sensing tests}

A bacterial suspension was employed to demonstrate the capability of an AAO-based parallel-plate capacitor act as an effective capacitive touch sensor. In standard molecular microbiological procedures, Lysogeny Broth (LB) (Grisp, Portugal) is used as liquid medium in qualitative procedures for the growth, cultivation and maintenance of recombinant Escherichia coli strains. In this work, the biological tests were only realized over the AAO structures fabricated from the anodization process using phosphoric acid electrolyte for $0.67 \mathrm{~min}$ (corresponding to the AAO sample coded as P1, according to Table 1). The reason why we selected the AAO - P1 sample to perform the biological tests is because this sample showed the best capacitive response arising from the contact with an $\mathrm{Al}$ metallic disk that covered the entire surface area of the anodized samples.

In order to perform biological tests, a pre-inoculum of $E$. coli BL21(DE3) was prepared in LB medium and the bacterial culture was grown overnight at $37^{\circ} \mathrm{C}$ with constant stirring at $200 \mathrm{rpm}$. The initial optical density of the medium was adjusted to $\mathrm{OD}_{600 \mathrm{~nm}}=0.1$. In fact, it is important to emphasize that the medium optical density is directly related to the amount of $E$. coli present in the bacterial suspension. Then, the AAO-P1 samples, previously disinfected with increasing concentrations of ethanol, were fully dipped in the culture medium with E. coli and were incubated at $37{ }^{\circ} \mathrm{C}$ without agitation. Afterwards, the AAO-P1 samples were withdrawn from the bacterial culture medium at different time intervals, namely 1, 4 and 8 hours, and the sensors were coded as A1, A4 and A8, respectively. These time intervals match the times for the $E$. coli growth and thus, it is expected that within these different time periods, the bacterial colony will multiply due to the conditions provided by the culture medium. Another sensor coded as AC, which is a control sensor, immersed only in the liquid LB medium without $E$. coli, was also considered. Then, the sensors were subjected to a drying process in an oven at $40{ }^{\circ} \mathrm{C}$ for $1 \mathrm{~h}$, and subsequently connected to the LCR meter (QuadTech 1920 Precision LCR Meter) in order to record variations on their capacitive response resulting from changes in the nanotextured surface area of the Au top electrode due to the presence of $E$. coli. The experimental sequence is schematically shown in Fig. SI4 of ESI. $\dagger$ The performance of the AAO-based capacitive touch sensors was monitored via a capacitance-time plot and the AAO-P1 samples with and without $E$. coli were observed by scanning electron microscopy (SEM).

\subsection{Morphological characterization of AAO samples}

The morphology and thickness of all fabricated AAO structures were analysed by scanning electron microscopy (SEM) using a FEI Quanta 400FEG ESEM/EDAX Genesis X4M. The SEM micrographs were subsequently exported to a commercial image-processing program, ImageJ, in order to calculate the AAOs structural parameters, namely porosity, pore diameter, inter-pore distance and pore density.

\section{Results and discussion}

\subsection{Current-time characteristics and AAO morphology}

The Fig. 2(a) and (b) show the current-time characteristic behaviour of the AAO samples P1 and P2 fabricated under hard anodizing conditions, according to Table 1 .

The typical behaviour of $I(t)$ characteristic curves are welldescribed elsewhere. ${ }^{31-34}$ From Fig. 2(a) it is possible to distinguish three main regions (R1 and R2). Briefly, at an initial stage (region R1) the current intensity decreases rapidly with time since it is related to the formation of a thin aluminium oxide barrier layer, which should form immediately after the start of the anodizing process. ${ }^{31-34}$ 

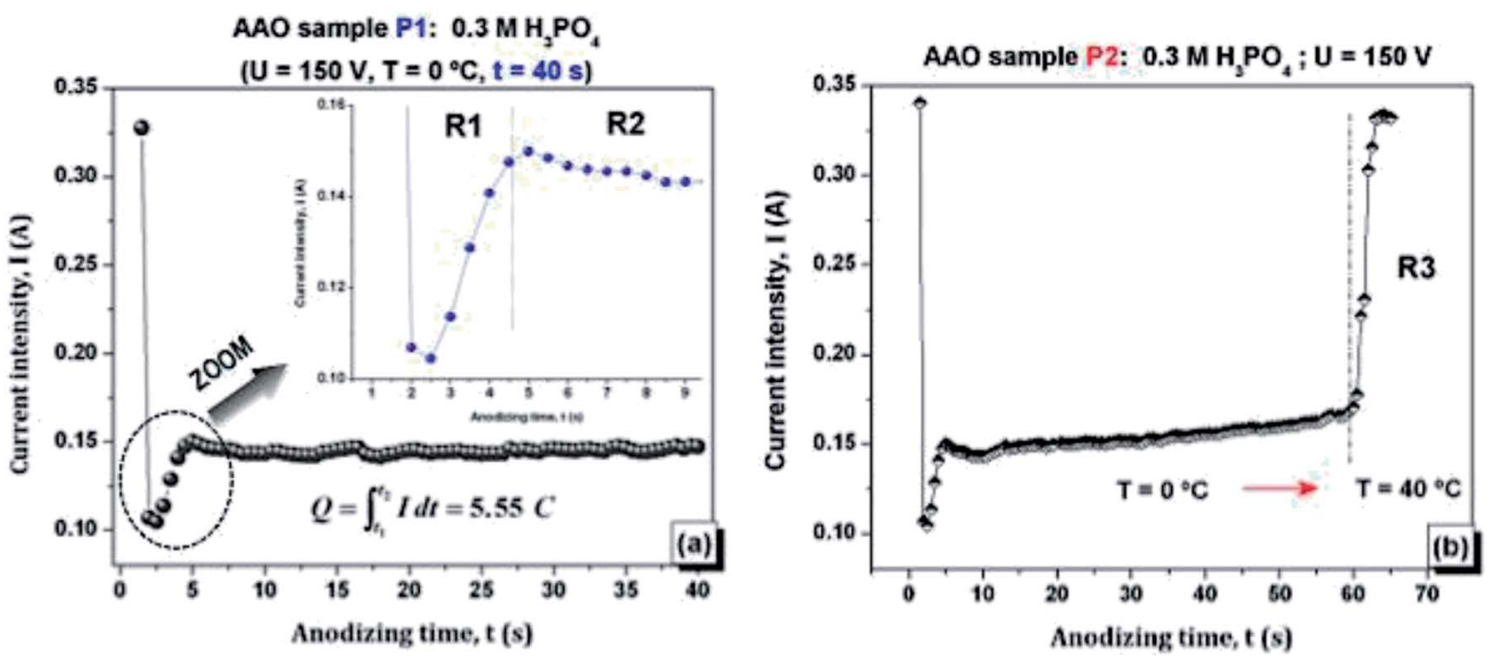

Fig. 2 Current-time plot related to anodized sample P1 and P2. (a) The current intensity for sample P1 shows two main regions - R1, showing a sharp drop in current intensity and region R2, with a steady current intensity; and (b) the current intensity for sample P2 behaved almost identical to the P1 for the first $60 \mathrm{~s}(t=60 \mathrm{~s})$. The main difference of the current profiles is that from $t=60 \mathrm{~s}$ (region R3) a sharp increase of the current intensity was observed for the sample P2.

The current intensity then increases slightly reaching a local maximum. At this stage, the barrier layer experiences a thinning process until it reaches a stable thickness and, simultaneously, the pore formation is initiated on the convex surface due to the increase of the local electric field. In region R2, the current intensity reaches a steady value, which is related to the growth of the porous layer.

On the other hand, from the current-time characteristic curve the thickness $h$ of the formed AAO structure can be estimated by applying the Faraday's law, ${ }^{35}$
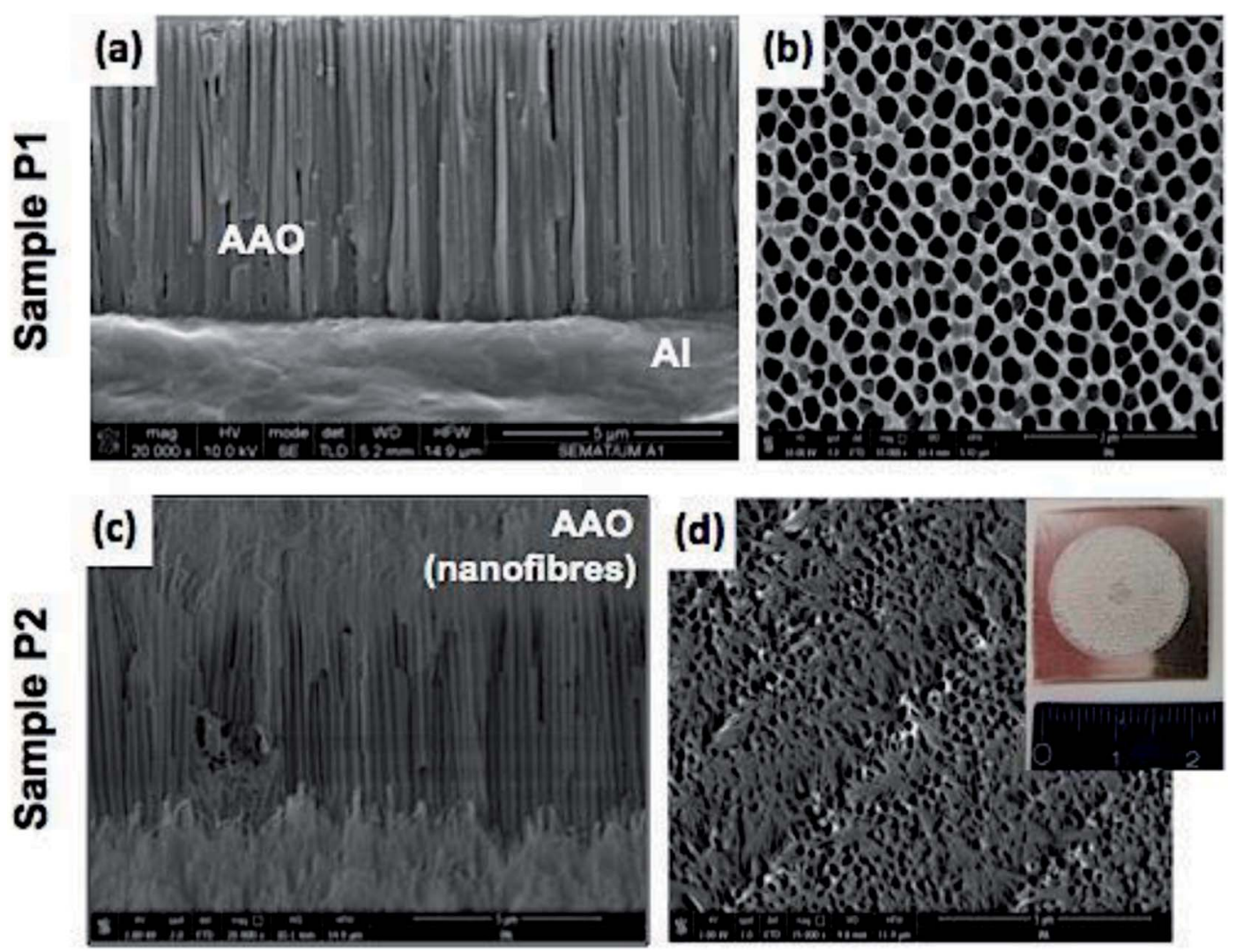

Fig. 3 SEM micrographs of AAO nanostructures fabricated under hard anodizing conditions; (a) and (b) are side and top view micrographs for sample P1, respectively whereas (c) and (d) are the side and top view micrographs for sample P2, respectively. 


$$
h=\frac{M Q}{z F \rho A}
$$

where $z$ is the number of electrons involved in the anodizing reaction (in the present case, 6 electrons), $F$ is the Faraday constant (96 $485 \mathrm{C} \mathrm{mo1}^{-1}$ ), $Q$ is the total electrical charge passing in the anodizing process and $M$ and $\rho$ are the molar mass and density of alumina, respectively. The total electrical charge $Q=\int_{t_{1}}^{t_{2}} I d t$ is the integral of current with respect to time, which can be calculated from the experimental $I(t)$ characteristic curve. The thickness of sample $\mathrm{P} 1$, determined by eqn (1), is about $5.54 \mu \mathrm{m}$, which is a reasonably value close to the one measured by SEM $(\approx 6.3 \mu \mathrm{m})$, as shown in Fig. 3(a), which refers to the side view micrograph of sample P1.

The analysis of SEM micrographs presented in Fig. 3(a) and (b) reveals that, in general, the sample P1 holds a morphology characterized by a well-defined and self-ordered porous alumina. The AAO structure of the sample P1 has an average nanopore diameter and inter-pore distance of $\sim 183 \mathrm{~nm}$ and $294.8 \mathrm{~nm}$, respectively.

$I(t)$ characteristic plot of sample P2, (Fig. 3(b)) shows that after $t=60 \mathrm{~s}$, there was a sharp increase in current intensity (region R3) resulting in a strong local temperature rise due to Joule heating. It has been pointed out that generated heat plays an important role in the enlargement of the pore size by assisting dissolution of the oxide from pore walls, resulting in truncated pore channels. ${ }^{36}$ This behaviour can be clearly confirmed by the observation of Fig. 3(c) and (d), which correspond to side and top view of the SEM micrographs for sample P2, respectively. The inset shown in Fig. 6(d) is a photographic image of the anodized surface for sample $\mathrm{P} 2$, which experienced a "local burning" due to Joule heating. It is possible to observe a damaged and quite irregular surface, which results from the collapse of the pores. It is important to note that although sample P2 does not appear to be useful for the main goal of this work, the destruction of its AAO structure due to pore collapse has led to the formation of alumina nanofibres, which may be of great interest to several other nanotech applications. The process reveals an efficient and economical method to produce $\mathrm{Al}_{2} \mathrm{O}_{3}$ nanofibres.

The SEM micrographs presented in Fig. 4(a-c) show the side view of the typical nanotextured Au-AAO-Al (MIM) parallel-plate capacitor referring to samples SU, OX and P1, respectively. The insets shown in Fig. 4(a) and (b) correspond to the SEM top view micrographs of non-textured Au anodized samples SU and OX, respectively (i.e. before thermal evaporation of gold). On the other hand, the inset shown in Fig. 4(c) is the photographic image of sample P1, which reveals the general appearance of its $\mathrm{Au}$ nanotextured surface area (Au top electrode).

Analysing SEM side view micrographs shown in Fig. 4(a-c) it can be concluded that, in general, for all the produced samples, the thermally evaporated gold was deposited homogeneously and mostly in the regions of the pore walls, thus making a nanoporous layer of $\mathrm{Au}$ on the surface of the AAO nanostructures. This evidence is attested by examining Fig. 4(c), which is the SEM micrograph of sample P1 acquired with backscattered electrons (BSE), therefore enabling to observe the typical atomic brightness contrast related with the existence of two different materials, namely alumina and gold. In addition, compared to AAO structures before the deposition of $\mathrm{Au}$ the average diameters of pores have been slightly reduced after the deposition of $\mathrm{Au}$, which can influence the electrical response of these AAO structures. According to Nielsch et al. ${ }^{37}$ the porosity $P$ (volume fraction of nanopores) of the AAO-based capacitive touch sensor can be expressed as,

$$
P=\frac{\pi}{2 \sqrt{3}}\left(\frac{D_{\mathrm{p}}}{D_{\text {int }}}\right)^{2}
$$

where $D_{\mathrm{p}}$ (in nm) and $D_{\text {int }}$ (in $\mathrm{nm}$ ) are the AAO pore diameter and the inter-pore distance, respectively, as indicated in Fig. 1(b). On the other hand, the AAO pore density, $n$, is defined as the total number of pores that occupy a surface area of $1 \mathrm{~cm}^{2}$, and can be given as,

$$
n=\frac{10^{14}}{\frac{\sqrt{3}}{2} D_{\text {int }}^{2}}
$$
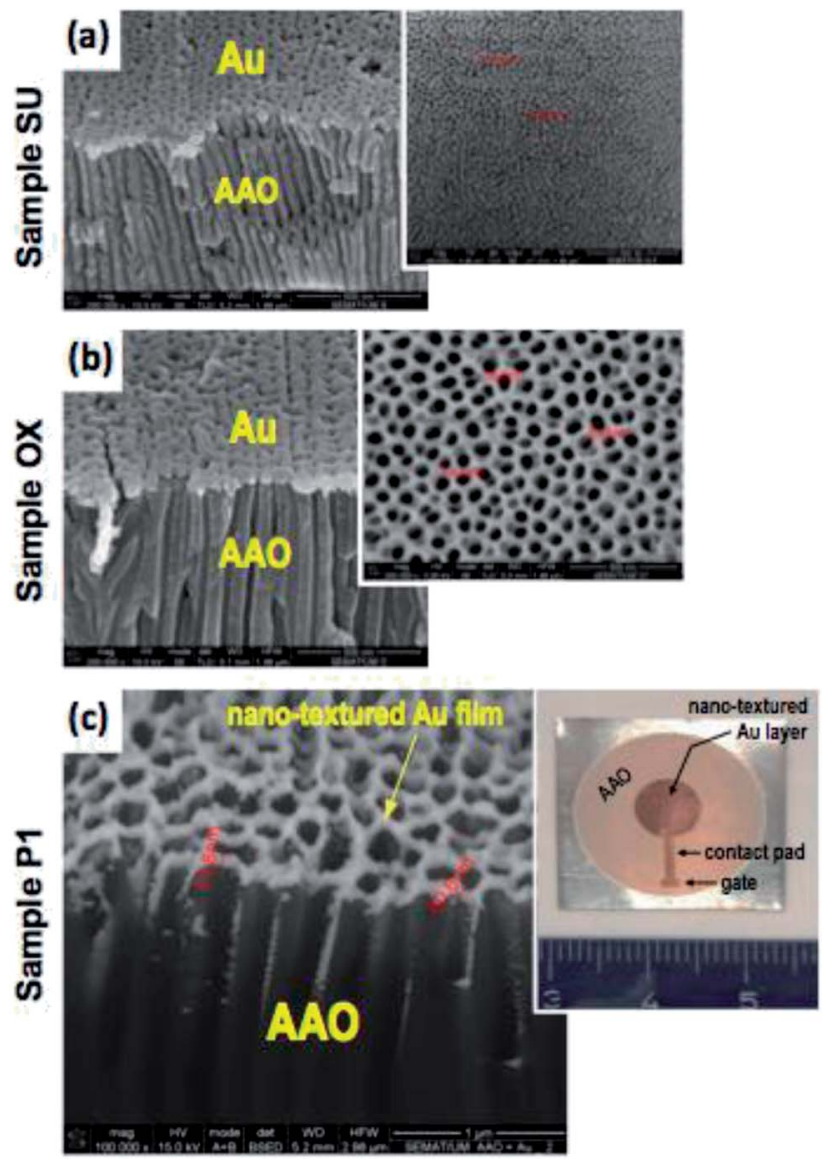

Fig. 4 Side view SEM micrographs of nanotextured MIM (Au-AAO-Al) parallel-plate capacitor produced with (a) sulphuric acid electrolyte (sample SU); (b) oxalic acid electrolyte (sample OX) and (c) phosphoric acid electrolyte (sample P1). The insets shown in (a) and (b) correspond to the SEM top view micrographs of non-textured $\mathrm{Au}$ anodized samples SU and OX, respectively. The inset shown in (c) is a photo of the surface of sample P1 showing the general appearance of its nanotextured Au top electrode. 
Table 2 Structural parameters for the fabricated AAO nanostructures

\begin{tabular}{lcccc}
\hline & $\begin{array}{l}\text { Pore diameter, } \\
D_{\mathrm{p}}(\mathrm{nm})\end{array}$ & $\begin{array}{l}\text { Inter-pore distance, } \\
D_{\text {int }}(\mathrm{nm})\end{array}$ & $\begin{array}{l}\text { Calculated porosity, } \\
P(\%)\end{array}$ & $\begin{array}{l}\text { Calculated pore } \\
\text { density, } n\left(\text { pore } / \mathrm{cm}^{2}\right)\end{array}$ \\
\hline SU & 21.8 & 45.2 & 21.0 & $5.6 \times 10^{10}$ \\
OX & 58.0 & 118.7 & 22.0 & $8.1 \times 10^{9}$ \\
P1 & 183.0 & 294.8 & 35.0 & $1.3 \times 10^{9}$
\end{tabular}

In order to evaluate AAO structural parameters, a statistical analysis was performed over the top view SEM micrographs presented in Fig. 3 and 4 by using the image-processing program, ImageJ. Table 2 show the structural dimensions for the produced AAO nanostructures.

Table 2 does not include results of the structural parameters for sample P2, since the morphology of this sample does not present self-organized pores due to the almost complete destruction of its nanoporous structure arising from the collapse of pore walls. For the other samples, the results shown in Table 2 reveal that the structural parameters of AAO samples are strongly dependent on the anodizing conditions (i.e. hard or mild conditions). For example, concerning the sample P1, fabricated with $0.3 \mathrm{M}$ phosphoric acid electrolyte $(U=150 \mathrm{~V}$ and $T=0{ }^{\circ} \mathrm{C}$ ), the values of its structural parameters, namely $D_{\mathrm{p}}, D_{\text {int }}$ and $P$ are about 8.4, 6.5 and 1.6 times higher than those the obtained for sample SU. As a consequence, sample P1 has a pore density that is markedly smaller (about 43 times lower) than that of the SU sample.

\subsection{Capacitive characteristics of the AAO capacitive sensor}

The capacitances of the fabricated AAO-based parallel-plate capacitors were estimated with the help of an LCR meter (QuadTech 1920 Precision LCR Meter), under a $100 \mathrm{kHz}$ input signal with an amplitude of $1.0 \mathrm{~V}$. This frequency was chosen because it is the one for which we obtained the lowest material dielectric losses (below 1\%). The dielectric constant of the AAO material $\left(\varepsilon_{\mathrm{AAO}}\right)$ can be expressed as,

$$
\varepsilon_{\mathrm{AAO}}=\frac{C \times d}{\varepsilon_{0} \times A_{\mathrm{G}}}
$$

where $d$ is the AAO thickness, $\varepsilon_{0}$ is the permittivity in the air, $C$ is the measured capacitance of the AAO capacitive sensor and $A_{\mathrm{G}}$ is the AAO surface area covered by the Au film, which can be expressed as $A_{\mathrm{G}}=A(1-P)$, where $A$ is the circular area of the flat surface without nanopores.

Fig. 5 shows the capacitance variations, $\Delta C$ (from which the sensors' sensitivity was calculated) for AAO sensors as the Au top detection electrodes come into contact with a metal $\mathrm{Al}$ circular disk with $7.5 \mathrm{~mm}$ in diameter (inset).

Noteworthy is that the initial capacitance is the capacitance of the AAO sensor with open pores but not covered by the metal Al circular disk. Thus, the greater the deviation of the capacitance from the AAO sensor initial capacitance, higher the device sensitivity is.

For example, considering the measured capacitance for sample P1 and applying eqn (4), the dielectric constant $\left(\varepsilon_{\mathrm{AAO}}\right)$ can be estimated to be 8.69 , which is close to the values typically reported in literature. ${ }^{38,39}$ In this work, the sensitivity, $S$, of the

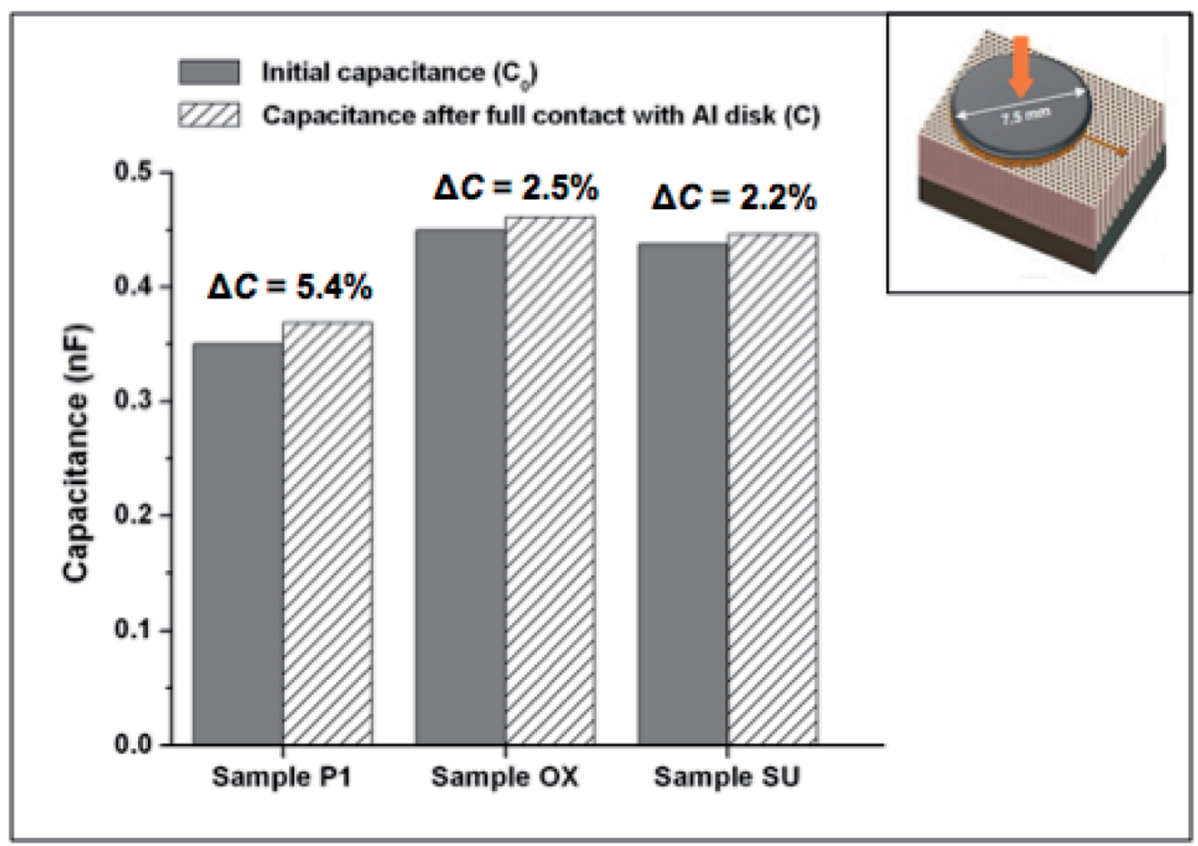

Fig. 5 The initial measured capacitance and the capacitance after the full contact of an Al disk with the Au nanotextured top electrode of the AAO capacitive sensor. 
AAO-based capacitive touch sensors in contact with the metal Al circular disk was determined using eqn $(5)^{\mathbf{4 0}}$

$$
S=\left(\frac{\Delta C \%}{A}\right)
$$

where $\Delta C \%=\left[\left(C-C_{0}\right) / C_{0}\right] \times 100$ is the percentage change in capacitance of the sensor in which $C$ corresponds to the capacitance value of the AAO sensor in contact with the metal Al circular disk, $C_{0}$ is the initial capacitance of the AAO sensor (with open pores but not covered by the metal Al circular disk) and $A$ is the top sensing electrode area fully contacted with the Al conductor circular disk of $7.5 \mathrm{~mm}$ in diameter. From Fig. 5, it is possible to observe that P1 sample (anodized with phosphoric acid electrolyte) present a capacitance change of 5.4\%, which is the highest value for the different produced samples. Moreover, by using eqn (5), it was also observed that the AAO sensor with the average highest sensitivity of $0.12 \Delta C \% / \mathrm{mm}^{2}$ corresponds to P1 sample (anodized with phosphoric acid electrolyte) while the other two types of AAO sensors fabricated under mild anodizing conditions, (namely the sample OX and sample SU) showed
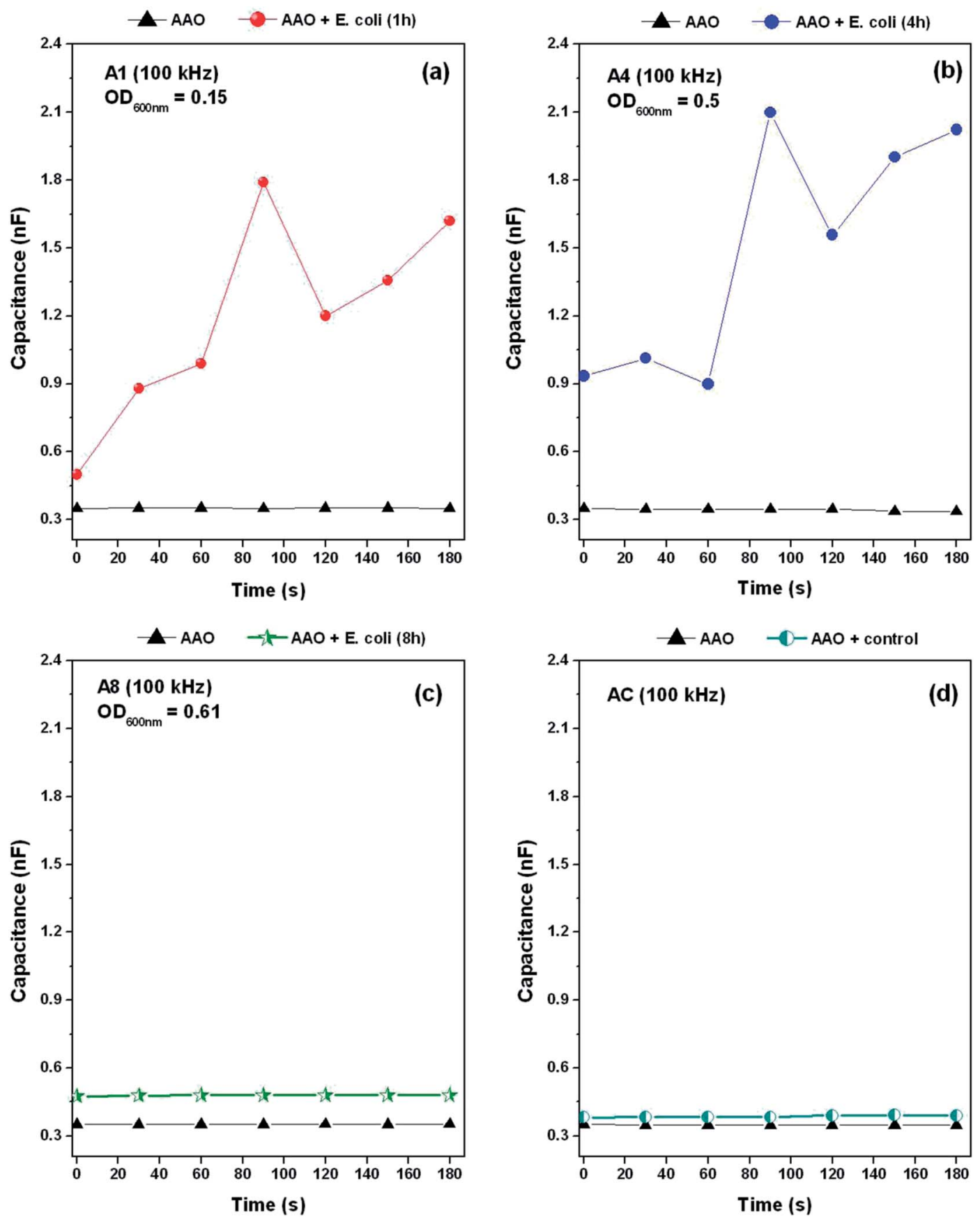

Fig. 6 Measurement of the capacitance variation with time for the AAO touch sensor due to the contact with a suspension of Escherichia coli bacteria. The values were recorded from (a) sample A1 (E. coli grown for $1 \mathrm{~h}$ ) (b) sample A4 (E. coli grown for 4 h) (c) sample A8 (E. coli grown for 8 h) and (d) control sample AC that does not contain E. coli as it was only immersed in the LB liquid medium. The triangular dots (identified as AAO) correspond to the recorded signal from the clean sensor (i.e. without liquid medium and $E$. coli), which is used as reference. 
much lower sensitivities of $0.06 \Delta C \% / \mathrm{mm}^{2}$ and $0.05 \Delta C \% / \mathrm{mm}^{2}$, respectively. This difference in sensitivities demonstrates that the AAO sensor showing a higher porosity (higher pore diameter) is more sensitive and therefore, more suitable for capacitive sensing/touch applications. When comparing the sensing performance of the present sensors with existing literature information on similar studies, it can be observed that the values of capacitance change obtained herein are in line with those assigned by Hong et al. ${ }^{29}$ In their work, the authors have produced AAO nanostructures by using a two-step anodizing conditions from an aqueous solution of $0.3 \mathrm{M}$ oxalic acid at a voltage of $47 \mathrm{~V} \mathrm{DC}$, and have registered a capacitance change of about $4 \%$ (in average), thus obtaining an AAO sensor with sensitivity of $0.21 \Delta C \% / \mathrm{mm}^{2}$.

In fact, as the $\mathrm{Al}$ circular disk makes contact with the nanotextured Au film, AAO nanopores (containing air inside) will be covered and as a consequence, a variation in the sensor capacitance, $\Delta C_{\text {air, }}$ can be observed, as schematized in Fig. 1(c). If the surface of touch sensor is entirely covered by the Al disk (see Fig. 5), the theoretical change in the sensing area is $A \times P$ and the theoretical capacitance variation can be estimated as $\Delta C_{\text {air }}=\left[\varepsilon_{0} \varepsilon_{\text {air }}(A \times P) / d\right]$, where $\varepsilon_{\text {air }}$ is the dielectric constant of air. The sample $\mathrm{P} 1$ has the highest theoretical variation for sensor capacitance $\left(\Delta C_{\text {air }}=6.2 \%\right)$, which is a slightly higher value than the one experimentally obtained $(\Delta C=5.4 \%)$, thus suggesting the highest capacitance variation, could be chosen to evaluate the performance of the AAO capacitive sensor for bacterial sensing applications.

\subsection{Performance of the AAO sensor for bacterial sensing applications}

For a biological application, the AAO sensor was used to detect the presence over time of $E$. coli through micro-touch type sensing tests. Fig. $6(\mathrm{a}-\mathrm{c})$ shows the time variation of capacitance for the AAO touch sensor measured before (clean sensor) and after the contact with $E$. coli bacteria. On the other hand, Fig. 6(d) shows the variation with time of the capacitance for the control sensor (AC), which refers to the sample that was only immersed in the LB liquid medium (i.e. without the presence of E. coli). Triangular dotted lines used as reference in Fig. 6(a-c) denote the recorded signal from the clean sensor (i.e. with no bacterial suspension)

It can be observed that the capacitance of sensors has experienced great intermittent variations during the time interval in which the capacitance measurements were taken for the samples A1 and A4 (see Fig. 6(a) and (b), respectively). It is also noticeable that, in general, there is a tendency to an increase in the value of capacitance over time. The aforementioned figures also present the measured optical density values of the culture medium, $\mathrm{OD}_{600}=0.15$ and $\mathrm{OD}_{600}=0.50$ for samples $\mathrm{A} 1$ and $\mathrm{A} 4$, respectively. The values clearly show that the suspension used for sample A4 hold a much higher amount of bacteria than that of sample A1. In addition, this condition is also expressed by the values recorded for the capacitance at $t=0 \mathrm{~s}$, which are about $0.49 \mathrm{nF}$ and $0.93 \mathrm{nF}$ for sample $\mathrm{A} 1$ and A4, respectively.
In contrast, the sample A8, despite the existing amount of bacteria $\left(\mathrm{OD}_{600}=0.61\right)$ is higher than that for the previous two samples, it is surprising that the recorded values for capacitance remain constant during the measurement period, showing an average $\Delta C \%$ of about $35.7 \%$, which result from the increase in the measured capacitance when compared with the clean sensor. Furthermore, still keeping the comparison with the clean sensor, the AC control sample shows no significant changes in capacitance during the measurement time, as would be already expected.

After the stipulated incubation period, the AAO sensors were subjected to a drying process in an oven at $40{ }^{\circ} \mathrm{C}$ for $1 \mathrm{~h}$. Taking these experimental conditions and assuming a total evaporation of the LB liquid medium (the pores should only contain air inside), it is expected that E. coli has deposited on the nanotextured surface of the Au electrode, thus making the necessary electrical contact. Under these conditions, the AAO sensor's capacitance variation would result from the addition of new capacitors, connected in parallel, where the dielectric medium is air $\left(\varepsilon_{\text {air }}=1\right)$. In this case, the theoretical capacitance change is estimated as $6.2 \%$, as already mentioned. However, this value is far from the ones experimentally recorded. This observation suggests that some volume of the LB culture medium possibly remains inside the pores of the AAO structures, which can occur because the surface position of bacteria (covering the pores of the AAO nanostructures) could have avoided the evaporation of the LB liquid culture medium from the porous channels of the AAO nanostructure, since the temperature of $40{ }^{\circ} \mathrm{C}$ as well as samples exposed for $1 \mathrm{~h}$ inside the oven correspond to very moderate conditions. Under this situation, each individual pore would act (in a simplified arrangement) as two capacitors in series, where the effective dielectric constant has a contribution from the LB culture medium as well, as schematically shown in Fig. 7.

The total effective capacitance variation, $\Delta C$, can be determined as,

$$
\frac{1}{\Delta C}=\frac{1}{C_{1}}+\frac{1}{C_{2}}
$$

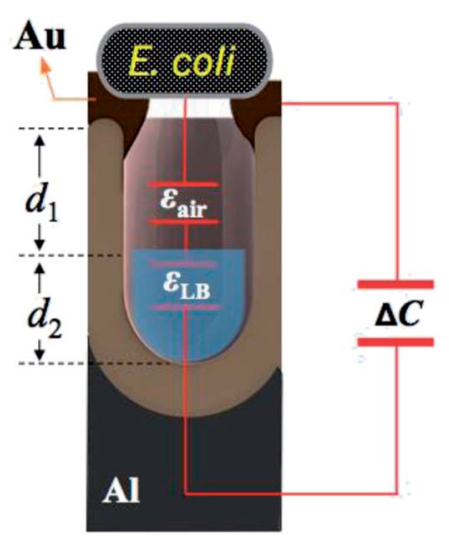

Fig. 7 Schematic for a single pore of an AAO-based capacitive sensor, where the volume inside is partially filled by air while the rest is filled by the LB culture medium. The capacitance variation $(\Delta C)$ results from the series connection of two capacitors with different dielectric constants ( $\varepsilon_{\text {air }}$ and $\left.\varepsilon_{\mathrm{LB}}\right)$ and thicknesses $\left(d_{1}\right.$ and $\left.d_{2}\right)$. 
where $C_{1}=\left[\varepsilon_{0} \varepsilon_{\text {air }}(A \times P) / d_{1}\right]$ is the capacitance of the first capacitor, which contains air as dielectric material with thickness $d_{1}$ while, $C_{2}=\left[\varepsilon_{0} \varepsilon_{\mathrm{LB}}(A \times P) / d_{2}\right]$ is the capacitance for the second capacitor with the dielectric of LB liquid medium with thickness $d_{2}$. For example, considering sample A8 and assuming that the dielectric constant of the LB culture medium, $\varepsilon_{\mathrm{LB}}$, is around the value of the dielectric constant of water, $\varepsilon_{\mathrm{LB}} \approx \varepsilon_{\text {water }}=78\left(20^{\circ} \mathrm{C}\right) .{ }^{41,42}$ According Fig. 6(c), the capacitance variation of about $35.7 \%$ for A8 sample (resulting from the increase on its capacitance) will be only achieved if the thickness of the LB culture medium is $d_{2} \approx 5.3 \mu \mathrm{m}$ and the air layer thickness is $d_{1} \approx 1 \mu \mathrm{m}$, which corresponds to about $84 \%$ and $16 \%$ of the total thickness $(6.3 \mu \mathrm{m})$ of the anodic layer of the A8 sample, respectively (see SEM micrograph in Fig. 3(a)). Moreover, as sample A8 was subjected to a long period of incubation, there was an increase on bacteria growth (number of bacteria) in comparison with the other points of time. For this incubation period, the higher number of bacteria could result in a strong reduction of bacteria mobility on the surface of the AAO-based sensor. As can be seen on Fig. 8(e) and (f) the E. coli bacteria formed a biofilm on the surface of the sensor thus reducing the mobility of individual bacteria. Under these conditions, characterized by the lack of bacteria mobility, the capacitance of the AAO sensor has effectively remained constant during measurements. These suggestions can be corroborated by the observation of the SEM micrographs shown in Fig. 8(a-f), which refers to the samples A1, A4 and A8.

For the case of $\mathrm{A} 1$ and $\mathrm{A} 4$ sensors, it is also possible to observe that the amount of bacteria was not sufficient to entirely

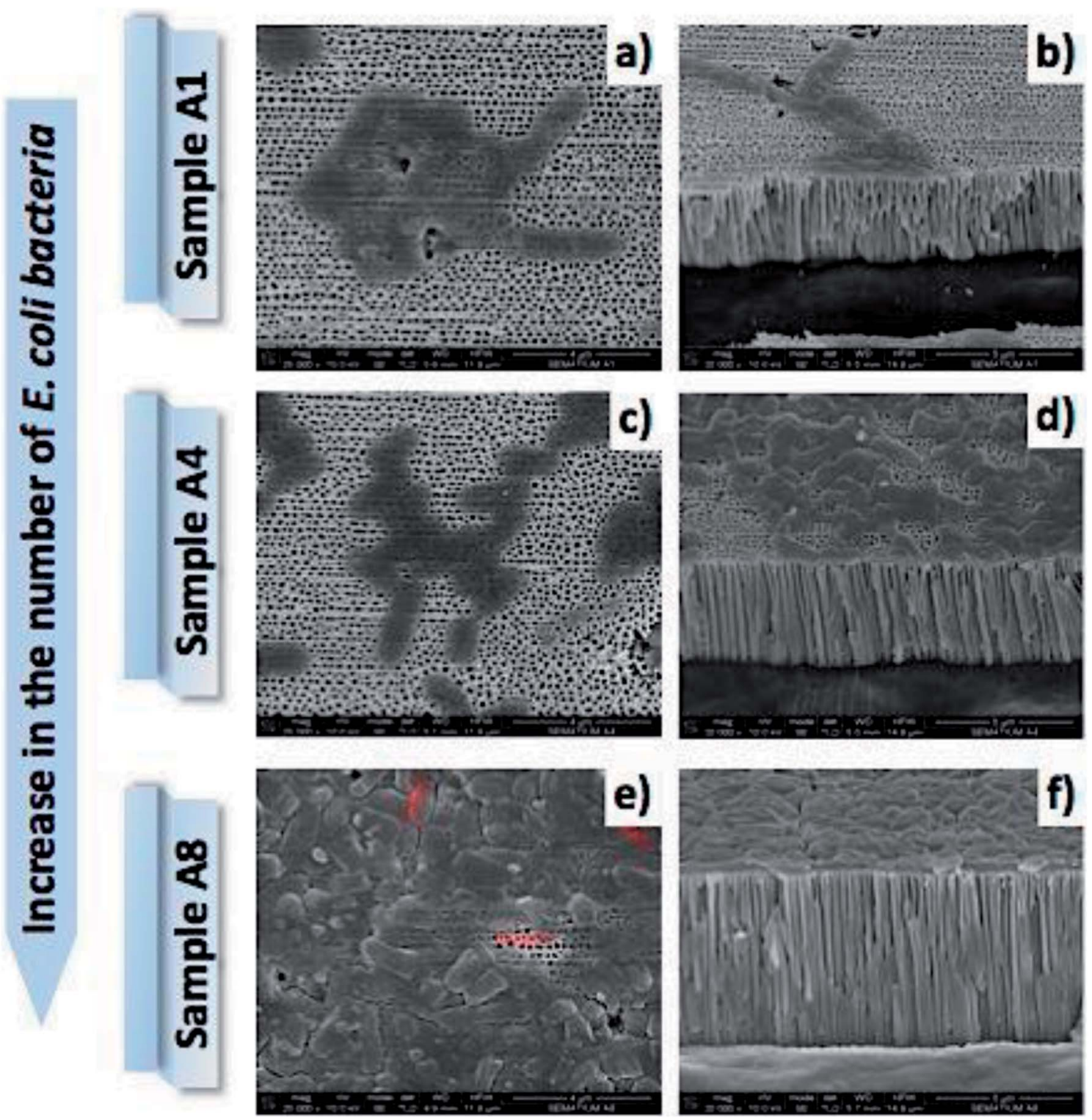

Fig. 8 SEM micrographs showing the $E$. coli cells located on the surface of the nanotextured Au electrode (deposited by thermal evaporation onto the nanoporous AAO structures). Micrographs (a), (c) and (e) are top view, and (b), (d) and (f) are side view micrographs of AAO-based sensors $A 1, A 4$ and $A 8$, respectively. 
cover the surface area of the Au electrode (see Fig. 8(a-d)). This finding confirms that the results shown in Fig. 6(a) and (b), revealing a very high increase in the capacitance of A1 and A4 samples, can not only be explained by the presence of bacteria on the surface of the Au electrode avoiding the evaporation of the LB liquid from the AAO nanoporous channels. It is anticipated that the moment the capacitance measurements were carried out, many bacteria would still be alive, presenting an adequate mobility to cause changes in the recorded capacitance. This assumption is justified because, as the population of the bacterial colony is relatively low (due to the moderately low incubation time for samples $\mathrm{A} 1$ and $\mathrm{A} 4$, respectively), as Semenov et al. reported, ${ }^{\mathbf{4 3}}$ there could be a weak competition between them in order to consume the "food" that is provided by a certain volume of the LB culture medium still enclosed in the pores of the AAO structure. Although this is a not a widely investigated topic, there is strong evidence that living microorganisms that make up a bacterial colony holds specific communication mechanisms for their cooperation and survival in community. ${ }^{\mathbf{4 4}}$ The work developed by Maxim ${ }^{\mathbf{4 4}}$ proves that one of the communication mechanisms used by bacteria is associated with the emission of infrared radiation (IR). In this context, we speculate that there is the possibility that the IR radiation frequency, emitted by the bacteria resting on the surface of the AAO structures, may be capable of selectively exciting determined vibration eigenmodes (and/or to cause some localized temperature changes), thus contributing to significant changes in the electrical properties of the AAO material or its surroundings. Nevertheless, this speculation needs further scientific investigation.

\section{Conclusions}

Nanoporous anodic aluminium oxides were successfully produced from three different types of electrolytes, namely sulphuric acid, oxalic acid and phosphoric acid by using a cylindrical homemade anodization cell made from a solid polycarbonate billet via a lathe machining process. Depending on the anodizing conditions, different AAO morphologies (pore size, inter-pore distance and porosity) were achieved and it was demonstrated that they influence the sensitivity and performance of capacitive touch sensors based on the manufactured AAO structures. The experimental results show that the best capacitive response for the AAO-based capacitive touch sensor was attained by using specific anodizing conditions requiring the use of $0.3 \mathrm{M}$ phosphoric acid at $150 \mathrm{~V}, 0{ }^{\circ} \mathrm{C}$ and a very fast anodizing process $(\sim 40 \mathrm{~s})$, because the initial capacitance for this Au-AAO-Al parallel-plate MIM capacitor (P1 sample) has been increased by $5.4 \%$ and its corresponding sensitivity was of $0.12 \Delta C \% / \mathrm{mm}^{2}$. This is a much higher value than the ones recorded for the other two sensors, namely the OX sample and SU sample, which presented sensitivity values of $0.06 \Delta C \% / \mathrm{mm}^{2}$ and $0.05 \Delta C \% / \mathrm{mm}^{2}$, respectively.

As a biological application, the AAO-based capacitive touch sensor has been successfully implemented to detect the presence and progress over time of a given amount of $E$. coli bacteria in contact with the Au nanotextured surface of the AAO-based sensor. It was found that depending on the amount of bacteria present on the Au nanotextured surface of the AAObased sensor, its capacitive response also varied, indicating the occurrence of different interaction mechanisms between the culture of bacteria and the sensor.

\section{Conflicts of interest}

There are no conflicts to declare.

\section{Acknowledgements}

This study was supported by the Portuguese Fundação para a Ciência e a Tecnologia (FCT) under the scope of the strategic funding of UID/FIS/04650/2013 and UID/BIO/04469/2013 units and COMPETE 2020 (POCI-01-0145-FEDER-006684). Artur Ribeiro thanks FCT for funding the scholarship with the reference SFRH/BPD/98388/2013.

\section{References}

1 H. Buff, Über das electrische Verhalten des Aluminiums, Liebigs Ann. Chem., 1857, 102(2006), 265-284.

2 P. G. Sheasby and R. Pinner, The Surface Treatment and Finishing of Aluminium and Its Alloys, 6th edn, Finishing Publications Ltd. \& ASM International, Materials Park, OH, and Stevenage, UK, 2001.

3 H. Masuda and K. Fukuda, Ordered Metal Nanohole Arrays Made by a Two Step Replication of Honeycomb Structures of Anodic Alumina, Science, 1995, 268(5216), 1466-1468.

$4 \mathrm{H}$. Masuda and M. Satoh, Fabrication of Gold Nanodot Array Using Anodic Porous Alumina as an Evaporation Mask, Jpn. J. Appl. Phys., 1996, 35(Part 2, No. 1B), L126-L129.

5 D. H. Park, H. Seung Kim, M.-Y. Jeong, Y. Baek Lee, H.-J. Kim, D.-C. Kim, J. Kim and J. Jo, Significantly Enhanced Photoluminescence of Doped Polymer-Metal Hybrid Nanotubes, Adv. Funct. Mater., 2008, 18(17), 25262534.

6 Ki H. Young, D. Hyuk Park, S. Kyu Park, H. Song, D.-C. Kim, J. Kim, H. H. Young, O. Kyung Park, B. Cheol Lee and J. Joo, Tuning and Enhancing Photoluminescence of Light-Emitting Polymer Nanotubes through Electron-Beam Irradiation, Adv. Funct. Mater., 2009, 19(4), 567-572.

7 Y. K. Hong, D. H. Park, S. Gi Jo, M. H. Koo, D. -C. Kim, J. Kim, J. -S. Kim, S. -Y. Jang and J. Joo, Fine Characteristics Tailoring of Organic and Inorganic Nanowires Using Focused Electron-Beam Irradiation, Angew. Chem., Int. Ed., 2011, 50(16), 3734-3738.

8 T.-C. Chan, Y.-M. Lin, H.-W. Tsai and Z. M. Wang, ChienNeng Liao, Yu-Lun Chueh, Growth of large-scale nanotwinned $\mathrm{Cu}$ nanowire arrays from anodic aluminum oxide membrane by electrochemical deposition process: controllable nanotwin density and growth orientation with enhanced electrical endurance performance, Nanoscale, 2014, 6, 7332-7338. 
9 S. Ghosh, T. Maiyalagan and R. N. Basu, Nanostructured conducting polymers for energy applications: towards a sustainable platform, Nanoscale, 2016, 8, 6921-6947.

10 U. Khan, M. Irfan, W. J. Li, N. Adeela, P. Liu, Q. T. Zhang and X. F. Han, Diameter-dependent multiferroic functionality in hybrid core/shell NWs, Nanoscale, 2016, 8, 14956-14964.

11 H. Wang, T. Chang, X. Li, W. Zhang, Z. Hu and A. M. Jonas, Scaled down glass transition temperature in confined polymer nanofibers, Nanoscale, 2016, 8(32), 14950-14955.

12 A. Khan Kasi, J. Khan Kasi, M. Hasan, N. Afzulpurkar, S. Pratontep, S. Porntheeraphat and P. Apirak, Fabrication of low cost anodic aluminum oxide (AAO) tubular membrane and their application for hemodialysis, $A d v$. Mater. Res., 2012, 550-553, 2040-2045.

13 A. Belwalkar, E. Grasing, W. Van Geertruyden, Z. Huang and W. Z. Misiolek, Effect of Processing Parameters on Pore Structure and Thickness of Anodic Aluminum Oxide (AAO) Tubular Membranes, J. Membr. Sci., 2008, 319(1-2), 192-198.

14 G. Gorokh, A. Mozalev, D. Solovei, V. Khatko, E. Llobet and $\mathrm{X}$. Correig, Anodic formation of low-aspect-ratio porous alumina films for metal-oxide sensor application, Electrochim. Acta, 2006, 52(4), 1771-1780.

15 E. P. Briggs, A. R. Walpole, P. R. Wilshaw, M. Karlsson and E. Pålsgård, Formation of highly adherent nano-porous alumina on Ti-based substrates: a novel bone implant coating, J. Mater. Sci.: Mater. Med., 2004, 15(9), 1021-1029.

16 M. Darder, P. Aranda, M. Hernández-Vélez, E. Manova and E. Ruiz-Hitzky, Encapsulation of enzymes in alumina membrane of controlled pore size, Thin Solid Films, 2006, 495(1), 321-326.

17 D. Gong, V. Yadavalli, M. Paulose, M. Pishko and A. Craig, Grimes, Controlled Molecular Release Using Nanoporous Alumina Capsules. Therapeutic Micro and Nanotechnology, Biomed. Microdevices, 2003, 5, 75-80.

18 J. Chen, S. Wang, L. Ding, Y. Jiang and H. Wang, Performance of through-hole anodic aluminum oxide membrane as a separator for lithium-ion battery, J. Membr. Sci., 2014, 461, 22-27.

19 Y.-keon Ahn, J. Park, D. Shin, S. Cho, Si Y. Park, H. Kim, Y. Piao, J. Yoo and Y. S. Kim, Enhanced electrochemical capabilities of lithium ion batteries by structurally ideal AAO separator, J. Mater. Chem. A, 2015, 3, 10715-10719.

20 Y. Gao, Y. Lin, J. Chen, Q. Lin, Y. Wu, W. Su, W. Wang and Z. Fan, Three-dimensional nanotube electrode arrays for hierarchical tubular structured high-performance pseudocapacitors, Nanoscale, 2016, 8, 13280-13287.

21 A. P. Samantilleke, J. O. Carneiro, P. Alpuim, V. Teixeira and T. T. Thu Thuy, Nanoporous alumina templates: anodisation and mechanical characterisation, Nanotechnology, 2012, 4, 319-342.

22 W. Lee and S. J. Park, Porous anodic aluminum oxide: anodization and templated synthesis of functional nanostructures, Chem. Rev., 2014, 114(5), 7487-7556.

23 P. G. Sheasby and R. Pinner, The Surface Treatment and Finishing of Aluminum and Its Alloys, 6th edn, Finishing Publications Ltd. \& ASM International, Materials Park, OH, and Stevenage, UK, 2001.
24 W. Lee, R. Ji, U. Gösele and K. Nielsch, Fast fabrication of long-range ordered porous alumina membranes by hard Anodization, Nat. Mater., 2006, 5, 741-747.

25 Y. Li, M. Zheng, L. Ma and W. Shen, Fabrication of highly ordered nanoporous alumina films by stable high-field anodization, Nanotechnology, 2006, 17, 5101-5105.

26 S. Ono, M. Saito and H. Asoh, Self-Ordering of Anodic Porous Alumina Induced by Local Current Concentration: Burning, Electrochem. Solid-State Lett., 2004, 7(7), B21-B24.

27 H. K. Lee, S. I. Chang and E. Yoon, A flexible polymer tactile sensor: Fabrication and modular expandability for large area deployment, J. Microelectromech. Syst., 2006, 15(6), 16811686.

28 J. P. Bergstrom and T. Dong, Rapid Detection of E. coli Cells in Urine Samples using a Self-Capacitance Touchscreen Device, IEEE Engineering in Medicine and Biology Society Conference Proceedings, 2015, pp. 5545-5548.

29 C. Hong, L. Chu, W. Lai, A.-S. Chiang and W. Fang, Implementation of a New Capacitive Touch Sensor Using the Nanoporous Anodic Aluminum Oxide (np-AAO) Structure, IEEE Sens. J., 2011, 11(12), 3409-3415.

30 N. Idil, M. Hedström, A. Denizli and B. Mattiasson, Whole cell based microcontact imprinted capacitive biosensor for the detection of Escherichia coli, Biosens. Bioelectron., 2017, 87, 807-815.

31 A. P. Samantilleke, J. O. Carneiro, S. Azevedo, T. Thuy and V. Teixeira, Electrochemical Anodizing, Structural and Mechanical Characterization of Nanoporous Alumina Templates, J. Nano Res., 2013, 25, 77-89.

32 M. Mibus, C. Jensen, X. Hu, C. Knospe, M. L. Reed and G. Zangari, Dielectric breakdown and failure of anodic aluminum oxide films for electrowetting systems, J. Appl. Phys., 2013, 114(1), 014901.

33 G. K. Singh, A. A. Golovin and I. S. Aranson, Formation of Self-organized Nanoscale Porous Structures in Anodic Aluminum Oxide, Phys. Rev. B: Condens. Matter Mater. Phys., 2006, 73, 205422.

34 X. Zhao, S.-K. Seo, U.-J. Lee and K.-H. Lee, Controlled Electrochemical Dissolution of Anodic Aluminum Oxide for Preparation of Open-Through Pore Structures, J. Electrochem. Soc., 2007, 154(10), C553-C557.

35 F. C. Walsh, The Overall Rates of Electrode Reactions: Faraday's Laws of Electrolysis, Trans. Inst. Met. Finish., 1991, 69(4), 155-157.

36 J. S. L. Leach and P. Neufeld, Pore structure in anodic $\mathrm{Al} 2 \mathrm{O} 3$ films, Corros. Sci., 1969, 9(6), 413-421.

37 K. Nielsch, J. Choi, K. Schwirn and B. Ralf, Wehrspohn, Ulrich Gösele, Self-ordering Regimes of Porous Alumina: The 10 Porosity Rule, Nano Lett., 2002, 2(7), 677-680.

38 P. Lo, C.-C. Lee, W. Fang, Implementation of electrostatically controlled Fabry-Perot interferometer using nanoporous anodic aluminum oxide layer, Transducers 2013, Barcelona, Spain, 16-20 June 2013, pp. 677-680.

39 J. I. Sohn, Y.-S. Kim, C. Nam, B. K. Cho and S. Tae-Yeon, Fabrication of high-density arrays of individually isolated nanocapacitors using anodic aluminum oxide templates 
and carbon nanotubes, Appl. Phys. Lett., 2005, 87(12), 123115.

40 G. R. Suma, N. K. Subramanic, S. Sachhidananda and S. V. Satyanarayana, Siddaramaiah, Nanotechnology Enabled E.Coli Sensors: An Opto-Electronic Study, Mater. Today, 2017, 4, 11300-11304.

41 C. G. Malmberg and A. A. Maryott, Dielectric Constant of Water from $0 \mathrm{C}^{\circ}$ to $1000{ }^{\circ} \mathrm{C}$, J. Res. Natl. Bur. Stand., 1956, 56(1), 2641.

42 C. Ren, L. Zeng and M. M. Zhou, Preparation, Biochemical Analysis, and Structure Determination of the
Bromodomain, an Acetyl-Lysine Binding Domain, Methods Enzymol., 2016, 573, 321-343.

43 A. V. Semenov, A. H. C. van Bruggen, Leo van Overbeek, A. J. Termorshuizen and A. M. Semenov, Influence of temperature fluctuations on Escherichia coli O157:H7 and Salmonella enterica serovar Typhimurium in cow manure, FEMS Microbiol. Ecol., 2007, 60(3), 419-428.

44 V. T. Maxim, Light-mediated “conversation" among microorganisms, Microbiol. Res., 2004, 159, 1-10. 